\title{
CFD simulations of RTD of a strawberry pulp in a continuous ohmic heater
}

\author{
I. Castro, N. Reis, J.A. Teixeira, A. A. Vicente ${ }^{1}$ \\ Centro de Engenharia Biológica, Universidade do Minho, Campus de Gualtar, 4710-057 \\ Braga, Portugal. Phone: +351.253 .604400 ; Fax: +351.253 .678986$
}

\begin{abstract}
A pilot ohmic heater is to be tested for the continuous aseptic processing of strawberry pulps and jams. The hydrodynamics and the fluid residence time distribution (RTD) have been experimentally investigated for Newtonian and non-Newtonian fluids (water and an industrial strawberry pulp, respectively), for several inlet flow rates. The results were obtained using Computational Fluid Dynamics (CFD) with a user-defined function (UDF) description of the fluid phase (pulp). For all of the conditions tested the fluid phase is described using the laminar flow model. The results show that the RTD is affected by the inlet flow rate but not so significantly by the process temperature. Some shortcuts and dead zones were detected in the ohmic heater specially for Newtonian fluids. The heater behaves like a piston flow with longitudinal mixing.
\end{abstract}

Keywords: CFD, RTD, ohmic heating, strawberry pulp

\section{Introduction}

Ohmic heating is defined as a process where electric currents are passed through foods with the main purpose of heating them by internal energy generation (de Alwis and Fryer, 1990). It has recently gained new interest because the products obtained are of clearly superior quality than those processed by conventional technologies. This is mainly due to its ability to heat materials rapidly and uniformly leading to a less aggressive thermal treatment (which, otherwise, often leads to over processed volumes). Ohmic heating can be considered a High Temperature Short Time (HTST) aseptic process. The potential applications of this technique in food industry are very wide and include e.g. blanching, evaporation, dehydration, fermentation and pasteurisation. Especially interesting is the application of this technique to foods containing solid particles and/or being viscous fluids (e.g. fruit purees and fruit pulps).

The most critical property affecting ohmic heating rate is food electrical conductivity $(\sigma)$. Food electrical conductivity is affected by a large number of parameters such as temperature, ionic strength, free water (Lima and Sastry, 1999) material microstructure

\footnotetext{
${ }^{1}$ Author to whom correspondence should be adressed
} 
and solids content (Sastry and Palaniappan, 1992, Sastry, 1992). Solids content, in particular, is known to have a significant influence on the electrical properties of the slurry and may have a decisive effect on the performance of the heat treatment process (Sastry, 1992; Zang and Fryer, 1993; Sastry and Salengke, 1998). Likewise, for a given solids content, it is expected that solid particles' size and shape will induce significant changes in the electrical conductivity of the mixture (Castro et al. 2003).

Continuous food-sterilization processes involve the flow of solid-liquid mixtures, generally highly viscous and with non-Newtonian behaviour, in pipes and reactors. Prediction of particles passage time in the system is required for ensuring sterility and optimizing product quality. Sterilization of such flows is complex and it is necessary to ensure that each part of the material is sterile without any part being overcooked. The use of the distribution functions allows visualising the flow patterns inside the continuous ohmic heater, to calculate mixing efficiencies and axial dispersion. This is of critical importance when calculating the flow rate at which the pulp must be pumped through the ohmic heater in order to ensure that the fastest particle (be it a solid particle or a volume of the fluid) is subjected to the desired amount of heat in order to receive the adequate heat treatment.

\section{Materials and Methods}

\subsection{The continuous ohmic heater}

The ohmic heater (figure 1a) was designed for the continuous aseptic process of food products, especially fruit pulps. It has a volume of $2,2 \mathrm{~L}$ and operates at a maximum flow rate of $2 \mathrm{~L} / \mathrm{min}$. The temperature range of the industrial aseptic processing of fruit pulps is between 40 (pre-mixture temperature) and $90^{\circ} \mathrm{C}$.

\subsection{The CFD model}

Fluent ${ }^{\mathrm{TM}}$ 6.2.22 software was used to simulate flow patterns under steady-state flow conditions in the ohmic heater. A 3D axisymmetric geometry was created using Gambit $^{\mathrm{TM}}$ 2.1.6 and imported into the Fluent models. In every case the Reynolds number $(R e)$ was lower then 2000 , so laminar flow was considered.

The hexagonal grid consisted of 378,348 cells. This was a structured mesh with boundary layers in the walls which were imposed to decrease the cells spacing near the walls (figure 1b). The inlet velocities were defined on the Fluent boundary conditions panel. A flat velocity profile was considered having an axial component only.

The solutions were considered converged when the normalized residuals were below $1 \mathrm{x}$ $10^{-3}$. Most of the problems required less then 5,000 iterations for convergence. For Re above 400 some convergence problems occurred but they were solved by the change (diminution) of under-relaxation parameters. The discretization schemes to solve the couple equations were the standard method for pressure equation, SIMPLEC algorithm for pressure-velocity coupling and a first order model for the momentum equation. 

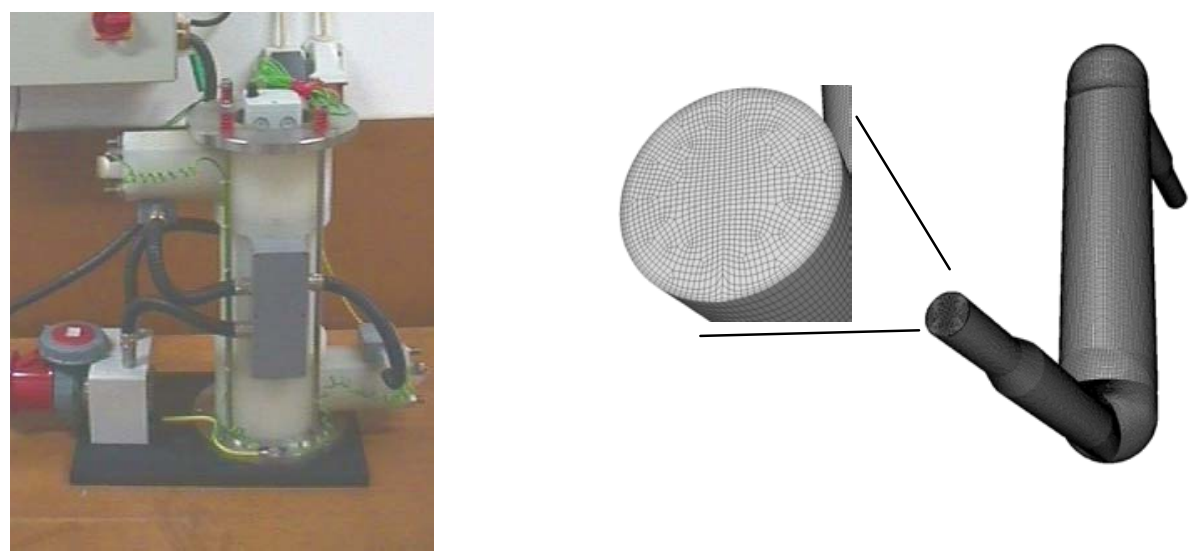

Figure 1 - a) ohmic heater; b) Mesh created for the ohmic heater; detail of the boundary layer.

\subsection{The RTD determination}

The simulated RTD were performed using an user defined scalar (UDS). Momentum, velocity and pressure equations were turned off and UDS value was determined at unsteady flow conditions. The scalar was initiated at a value of 1 at the inlet, and the outlet concentration was monitored by using an area weighted average.

\subsection{Determining the rheological properties of strawberry pulp}

The rheology of the strawberry pulp was determined using the New TA Instruments AR-2000 rheometer. A Peltier plate controlled Smart Swap temperature system was used along with a flat plate as the measuring system. A time controlled step flow procedure was used, applying successively higher shear rate values to the material. This procedure was repeated for eight different temperatures (ranging from 40 to $90{ }^{\circ} \mathrm{C}$ ).

$\eta=\frac{\tau_{0}+k\left[\gamma^{n}-\left(\frac{\tau_{0}}{\mu_{0}}\right)^{n}\right]}{\gamma}$

where,

$\tau_{0}$ - yield stress

$k$ - consistency index

$$
\begin{aligned}
& \mu_{0-\text { yielding viscosity }} \\
& \eta \text { - viscosity }
\end{aligned}
$$$$
\gamma \text { - shear rate }
$$$$
n \text { - power-law index }
$$

The obtained results fitted $\left(r^{2}>0,98\right)$ a Herschel-Buckley model (eq. 1). This model combines the effects of Bingham and power law in a fluid. For low strain rates $(\gamma<$ $\tau_{0} / \mu_{0}$ ) the material acts like a very viscous fluid with viscosity $\mu_{0}$. The increase of the strain rate increases the yield stress and the fluid behaves as a power law fluid. The Herschel-Buckley parameters were determined and are presented in table 1. 
Table 1 - Experimental parameters used to simulate pattern flows of strawberry

\begin{tabular}{cccc}
\hline Temperature & $\mathbf{4 0}^{\mathbf{}} \mathbf{C}$ & $\mathbf{6 0}^{\mathbf{}} \mathbf{C}$ & $\mathbf{9 0}^{\mathbf{}} \mathbf{C}$ \\
\hline $\boldsymbol{K}\left(\mathrm{kg} \cdot \mathrm{s}^{\mathrm{n}-2} \cdot \mathrm{m}^{-1}\right)$ & 0.08 & 0.42 & 0.20 \\
$\boldsymbol{n}(-)$ & 0.81 & 0.65 & 0.76 \\
$\tau_{\boldsymbol{0}}(\mathrm{Pa})$ & 1.48 & 5.19 & 9.42 \\
$\boldsymbol{\mu}_{\boldsymbol{0}}$ (Pa.s) & 0.32 & 1.38 & 1.01 \\
\hline
\end{tabular}

\section{Results and Discussion}

Simulations were performed for strawberry pulp for two different flow rates $(0.5$ and 2 $\mathrm{L} / \mathrm{min}$.) and three inlet temperatures $\left(40,60\right.$ and $\left.90{ }^{\circ} \mathrm{C}\right)$. The RTD results at the outlet are expressed in terms of the $F$-diagrams (expressed as the dimensionless outlet concentrations). In figure 2 such results are compared with both the ideal PF and CSTR behaviour (Danckwerts, 1952).

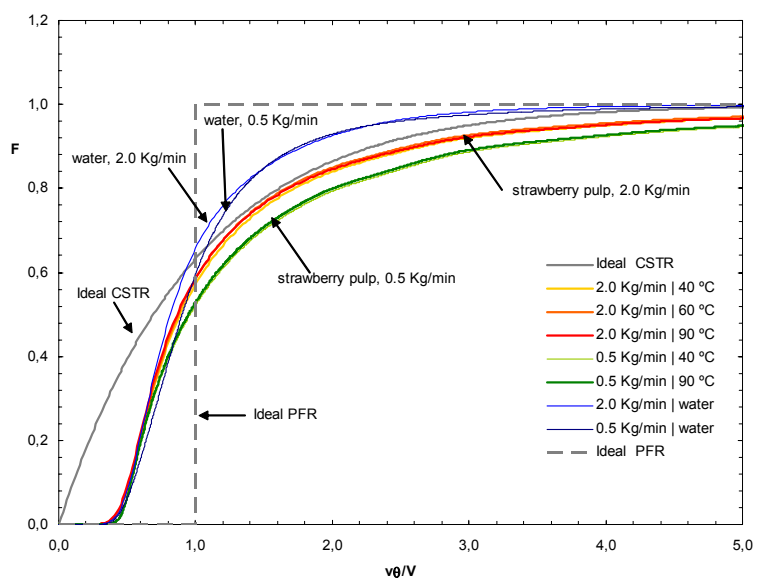

Figure 2- $F$ diagrams for strawberry pulp and water, at several inflows and temperatures ( $\mathrm{v}$ is the flow rate $\left[\mathrm{m}^{3} \cdot \mathrm{s}^{-1}\right], \theta$ is the flow time $[\mathrm{s}]$ and $\mathrm{V}$ is the ohmic heater volume $\left.\left[m^{3}\right]\right)$.

The hold-back $(H)$ is a parameter that measures the deviation from piston-flow (Danckwerts, 1953). $H$ varies from 0 for piston-flow to 1 when most of the reactor is occupied by dead zones; $H$ is equal to $1 / e(0.368)$ for a completely mixed vessel (CSTR). Segregation is a measurement of the efficiency of mixing and can be interpreted, in terms of the $F$-diagrams, as the departure from CSTR behaviour.

Table 2 - Hold back (H) and segregation (S) calculations for several conditions tested with strawberry pulp (v. $\tau / \mathrm{V}$ is the intersection with the CSTR F curve when $t=\theta$ )

\begin{tabular}{ccccccc} 
& \multicolumn{3}{c}{ Segregation } & \multicolumn{4}{c}{ Hold back } \\
\hline Flow rate & \multicolumn{2}{c}{ Water } & Water & \multicolumn{3}{c}{ Pulp temperature $\left({ }^{\mathbf{0}} \mathbf{C}\right)$} \\
$(\mathbf{L} / \mathbf{m i n})$ & $\boldsymbol{S}$ & $\boldsymbol{v} \cdot \boldsymbol{t} / \boldsymbol{V}$ & & $\mathbf{4 0}$ & $\mathbf{6 0}$ & $\mathbf{9 0}$ \\
$\mathbf{0 . 5}$ & 0.204 & 1.091 & 0.205 & 0.185 & 0.188 & 0.193 \\
$\mathbf{2 . 0}$ & 0.164 & 0.924 & 0.167 & 0.162 & - & 0.170 \\
\hline
\end{tabular}



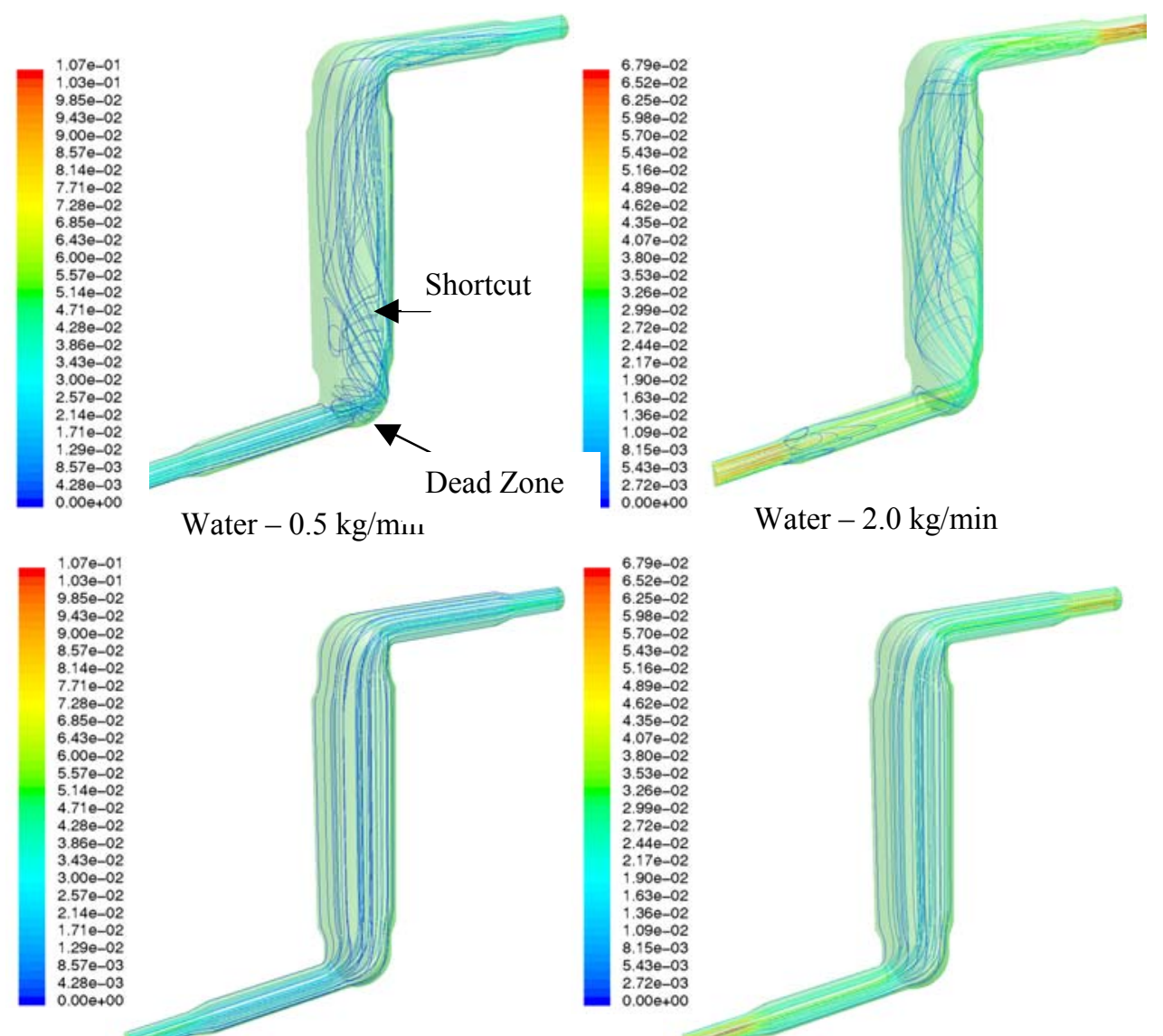

$.250-02$

$.43 e-02$

35e-02

44e-02

$2.720-03$

Strawberry pulp $-0.5 \mathrm{~kg} / \mathrm{min}, \mathrm{T}=40^{\circ} \mathrm{C}$

Strawberry pulp $-2.0 \mathrm{~kg} / \mathrm{min}, \mathrm{T}=40^{\circ} \mathrm{C}$
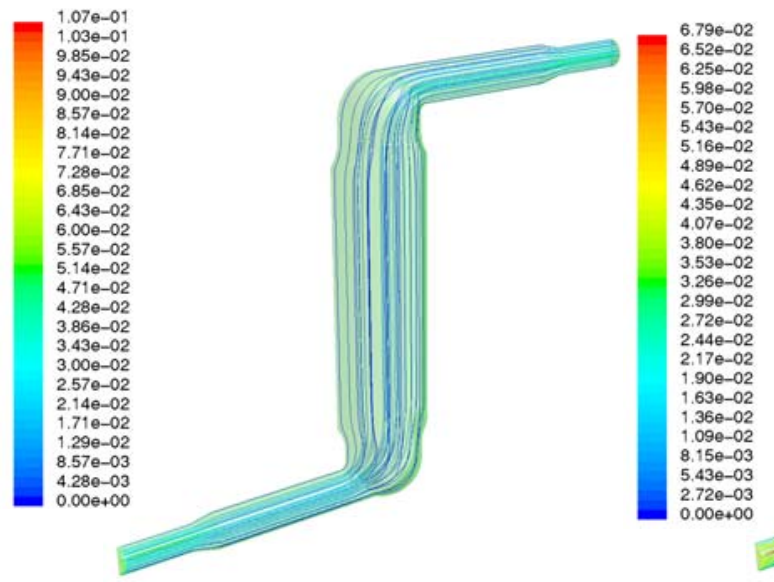

Strawberry pulp $-0.5 \mathrm{~kg} / \mathrm{min}, \mathrm{T}=90^{\circ} \mathrm{C} \quad$ Strawberry pulp $-2.0 \mathrm{~kg} / \mathrm{min}, \mathrm{T}=90{ }^{\circ} \mathrm{C}$

Figure 3- Simulated path lines, coloured by velocity magnitude values $(\mathrm{m} / \mathrm{s})$, for different fluids at different temperatures and flow conditions. 
By the analysis of table 2 and figures 2 and 3 it is possible to conclude, for strawberry pulp, that temperature does not affect significantly the flow dynamic behaviour of the heater when compared to the effect of the increase of inlet velocity. This causes a decrease of dead zones and shortcuts within the vessel. The decrease of shortcuts is related to the observed decrease of $H$ (Danckwerts, 1953) and the decrease of dead zones is related to the decreasing deviation from 1 of the values of $F$ for $(v \theta / V)=1$. For water the hydrodynamic behaviour is different, as seen from the flow lines in figure 3. In this case the values of segregation have been calculated and the flow rate seems to have a moderate influence in the behaviour of the heater. This is confirmed by the $F$ curves in figure 2.

\section{Conclusions}

Even though the ohmic heating process eliminates most of the conventional thermal gradients formed during heating, thus increasing the final product quality, the hydrodynamic behaviour of the heater could minimize this advantage. The increase of dead zones and shortcuts when using Newtonian fluids (such as water) may jeopardize the use of this ohmic heater for continuous sterilization of food products. In fact, the distribution of the residence times of the fluid means that the faster fraction will not be properly sterilized or the slowest will be over-processed. Thus the optimization of the heater should mainly be based on the hydrodynamic characteristics of the heater.

The deviation from plug behaviour of the flow was minimized when operating at high inlet velocities. The existence of shortcuts and dead zones affects the overall RTD but an optimisation of the heating process will be performed to overcome the design limitations of this ohmic heater.

\section{References}

Castro, I., Teixeira, J. A. and Vicente, A. A. (2003). The influence of field strength, sugar and solid content on electrical conductivity of strawberry products. J. Food Proc. Eng. 26, 17-29.

de Alwis, A.A., Fryer, P.J (1990). A finite-element analysis of heat generation and transfer during ohmic heating of food. Chem. Eng. Sci. 1547-155.

Danckwerts, P.V. (1953). Continuous flow systems- distribution of residence times. Chem Eng. Sci. 2, 1-13.

Lima, M. and Sastry, S.K. (1999). The effects of ohmic heating frequency on hot-air drying rate and juice yield. J Food Sci. 41, 115-119.

Sastry, S.K. and Palaniappan, S. (1992). Mathematical modeling and experimental studies on ohmic heating of liquid-particle mixtures in a static heater. J. Food Proc. Eng. 15, 241-261.

Sastry, S.K. (1992). A model for heating of liquid-particle mixtures in a continuous flow ohmic heater. J. Food Proc. Eng. 15, 263-278.

Sastry, S.K. and Salengke, S. (1998). Ohmic heating of solid-liquid mixtures: a comparison of mathematical models under worst-case heating conditions. J. Food Sci. 21, 441-458.

Zhang, L. and Fryer, P.J. (1993). Models for the electrical heating of solid-liquid food mixtures. Chem. Eng. Sci. 48, 633-642. 